\title{
Model to Hardware Matching for nm Scale Technologies
}

\author{
Sani Nassif
}

IBM

\begin{abstract}
Our ability to reliably predict the outcome of a semiconductor manufacturing process has been steadily deteriorating. This is happening because of two important factors.

- First, the overall CMOS technology slowdown has led to rapidly increasing complexity in the process and in its interaction with design. This has in turn caused an increase in the number and magnitude of systematic sources of mismatch between simulation models (both at the circuit simulation and timing levels) and hardware measurements.

- Second, manufacturing variability - long a source of concern only for analog design - is becoming important for digital designs as well and thus its prediction is now a first order priority. However, it is competing for the attention of researchers and CAD developers with a host of other so-called nm effects, thus slowing down the delivery of needed solutions.
\end{abstract}

The result is (a) our ability to arbitrarily compose a design out of disparate components is compromised because of a high degree of interaction between these components and (b) our ability to predict the nominal performance of a design as well as its tolerances and sensitivities is in danger. Phenomena like SRAM stability and leakage power variations are the first of many problems we are facing at $65 \mathrm{~nm}$ and below.

In this talk, we will review these issues and show how they are all related to the core issue of model to hardware matching. We will also show examples of potential solutions to this problem some of which are currently being developed in IBM, and some which are longer term and would benefit greatly from the attention of the academic community. 